\title{
Remitting Seronegative Symmetrical Synovitis with Pitting Edema Syndrome Complicated with Organizing Pneumonia
}

\author{
Chiaki Hosoda $^{1,2}$, Takashi Ishiguro ${ }^{1}$, Yasuhiro Morimoto ${ }^{1,2}$, Atsuki Furube ${ }^{1,2}$, Taisuke Isono ${ }^{1}$, \\ Yoshihiko Shimizu ${ }^{3}$ and Noboru Takayanagi ${ }^{1}$
}

\begin{abstract}
:
We herein report a 62-year-old man with idiopathic pulmonary fibrosis who developed remitting seronegative symmetrical synovitis with pitting edema (RS3PE) syndrome during follow-up. Pulmonary infiltrations were detected concomitantly with the development of RS3PE syndrome, and prednisolone improved both the pulmonary and extrapulmonary lesions. Recognizing the pulmonary manifestations of RS3PE syndrome is necessary to provide an appropriate diagnosis and disease management.
\end{abstract}

Key words: complication, idiopathic pulmonary fibrosis, pulmonary involvement, remitting seronegative symmetrical synovitis with pitting edema syndrome

(Intern Med 59: 1065-1069, 2020)

(DOI: 10.2169/internalmedicine.3713-19)

\section{Introduction}

Remitting seronegative symmetrical synovitis with pitting edema (RS3PE) syndrome is a rheumatic disease of elderly onset that was initially described by McCarty et al. in 1985 (1). It has been regarded as a distinct clinical entity characterized by symmetrical synovitis of the hands and ankles with pitting edema, elevated acute-phase reactants, and negative rheumatoid factor and has an excellent prognosis (2-6). There have been few reported cases of pulmonary complications of RS3PE syndrome $(7,8)$. It is important to recognize the variety of clinical presentations of RS3PE syndrome in order to provide an appropriate diagnosis and disease management.

We recently treated a man who developed RS3PE syndrome during follow-up of idiopathic pulmonary fibrosis (IPF). Consolidation on a background of lung fibrosis appeared concurrently with the onset of RS3PE syndrome, and these pulmonary lesions and the synovitis responded well to steroid treatment. We considered these pulmonary lesions to be a manifestation of RS3PE syndrome.

We herein report this case, focusing on the patient's pulmonary involvement and clinical course, in order to illustrate the varied clinical presentations of RS3PE syndrome.

\section{Case Report}

A 60-year-old asymptomatic man visited our hospital in May 2016 with abnormal shadows on chest X-ray found during a medical examination. He was an ex-smoker with a history of hypertension and obstructive sleep apnea syndrome. He had no history of rearing a pet, exposure to dust, or having symptoms suggestive of connective tissue diseases, such as arthralgia and dry mouth. Chest radiography revealed reticular shadows in both lower lung fields. Highresolution computed tomography (HRCT) showed subpleural reticular shadows and honeycomb formation in the basal area of both lungs, indicative of a usual interstitial pneumonia pattern (9). Autoimmune antibodies were negative, and we diagnosed him with IPF, for which he was followed without treatment.

\footnotetext{
${ }^{1}$ Department of Respiratory Medicine, Saitama Cardiovascular and Respiratory Center, Japan, ${ }^{2}$ Division of Respiratory Medicine, Department of Internal Medicine, The Jikei University School of Medicine, Japan and ${ }^{3}$ Department of Pathology, Saitama Cardiovascular and Respiratory Center, Japan

Received: July 25, 2019; Accepted: November 10, 2019; Advance Publication by J-STAGE: January 17, 2020

Correspondence to Dr. Chiaki Hosoda, ms03hosoda@jikei.ac.jp
} 


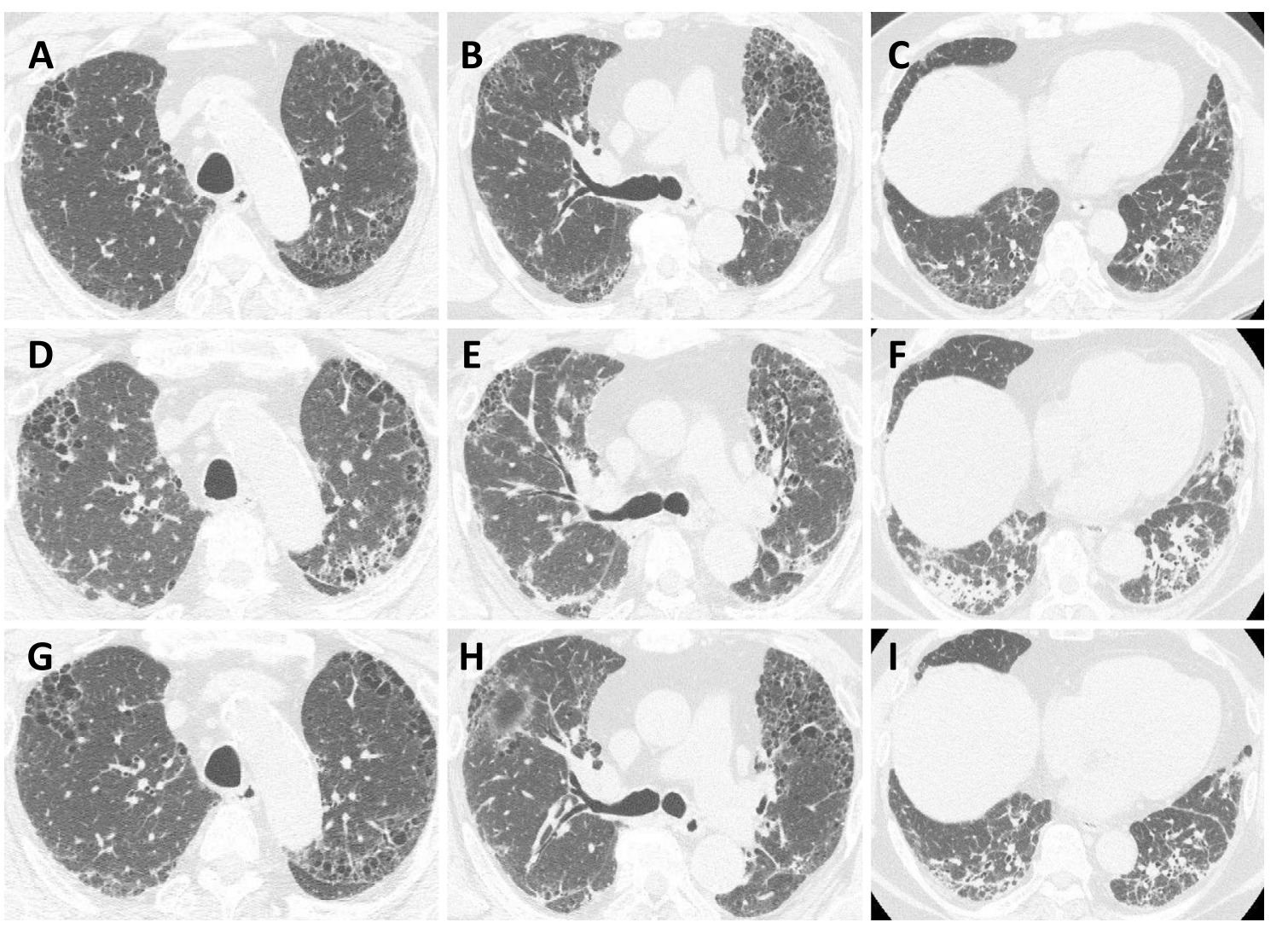

Figure 1. Chest high-resolution computed tomography (HRCT) findings. (A-C) Chest HRCT two weeks before admission showed a peripheral subpleural reticular shadow with honeycombing in the bilateral lung fields. (D-F) Chest HRCT on admission revealed consolidation on a background of lung fibrosis. (G-I) HRCT on hospital day 17 showed improvement of the consolidation.

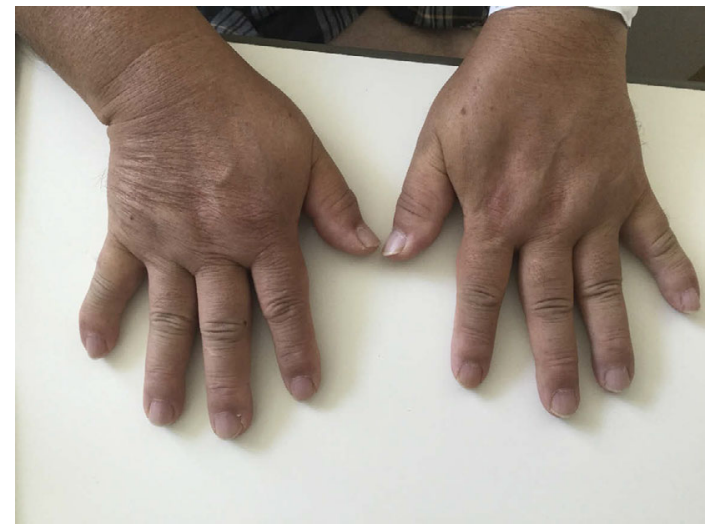

Figure 2. Photograph of the patient's hands taken on admission showing pitting edema of the dorsum of the hands along with swollen proximal interphalangeal joints and wrists.

On a regular follow-up examination in October 2018, there was almost no change in the chest HRCT and pulmonary function test findings (Fig. 1A-C). His white blood cell count of $9,000 / \mathrm{mm}^{3}$ and C-reactive protein (CRP) level of $0.78 \mathrm{mg} / \mathrm{dL}$ were not elevated. The level of Krebs von den Lungen-6 (KL-6) was $848 \mathrm{U} / \mathrm{mL}$. Serum matrix metalloproteinase-3 (MMP-3) was negative. Two weeks after this follow-up examination, he developed general fatigue, cough, and dyspnea on exertion, and arthralgia and pitting edema in his hands and feet appeared. These symptoms persisted for one week, during which his condition progressively worsened, so he was admitted to our hospital.

On admission, a physical examination revealed the following: respiratory rate, 28 breaths/min; heart rate, 82 beats/ min; blood pressure, $130 / 79 \mathrm{mmHg}$; and body temperature, $39.0{ }^{\circ} \mathrm{C}$. The examination further showed tender articular swelling and redness in his wrist, hand, knee, and foot joints with marked pitting edema at the bilateral dorsum of the hand, foot, and pretibial area (Fig. 2). Respiratory auscultation revealed fine crackles in the bilateral lower lung fields.

An arterial blood gas analysis under ambient air showed a partial pressure of oxygen of 63.1 Torr; partial pressure of carbon dioxide of 37.5 Torr; and $\mathrm{pH}$ of 7.46. Laboratory findings were as follows: white blood cell count, $11,000 / \mathrm{mm}^{3}$; neutrophil count, 9,200/ $\mathrm{mm}^{3}$; hemoglobin, 16.3 $\mathrm{g} / \mathrm{dL}$; platelets, $47.0 \times 10^{4} / \mathrm{mm}^{3}$; serum total protein, $7.3 \mathrm{~g} / \mathrm{dL}$; albumin, $3.4 \mathrm{~g} / \mathrm{dL}$; creatinine, $0.83 \mathrm{mg} / \mathrm{dL}$; lactate dehydrogenase, $162 \mathrm{IU} / \mathrm{L}$; CRP, $7.27 \mathrm{mg} / \mathrm{dL}$; KL-6, 819 U/mL; erythrocyte sedimentation rate, $37 \mathrm{~mm} / \mathrm{h}$; and brain natriuretic peptide, $<5.8 \mathrm{pg} / \mathrm{mL}$. The serum MMP-3 level was elevated to $230 \mathrm{ng} / \mathrm{mL}$ (normal: $<121 \mathrm{ng} / \mathrm{mL}$ ). Rheumatoid factor, anti-Ro/SSA antibody, anti-La/SSB antibody, antiscleroderma 70 antibody, and anti-cyclic citrullinated peptide antibody were all negative, as were antibodies for HIV, hepatitis B surface antigen, and anti-hepatitis $\mathrm{C}$ virus. 


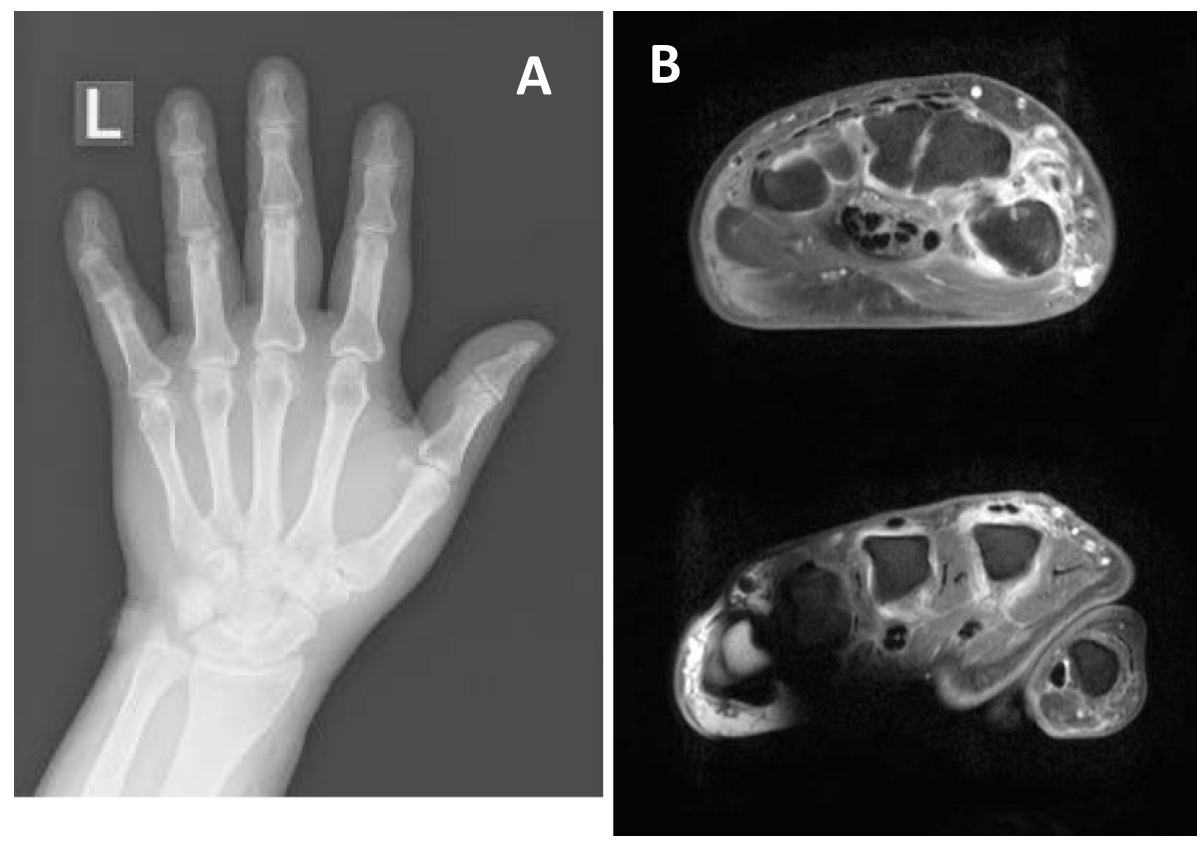

Figure 3. X-ray (A) and gadolinium contrast-enhanced dynamic magnetic resonance imaging (B) of the patient's hands showing soft tissue swelling and extensor tenosynovitis without bone erosions.
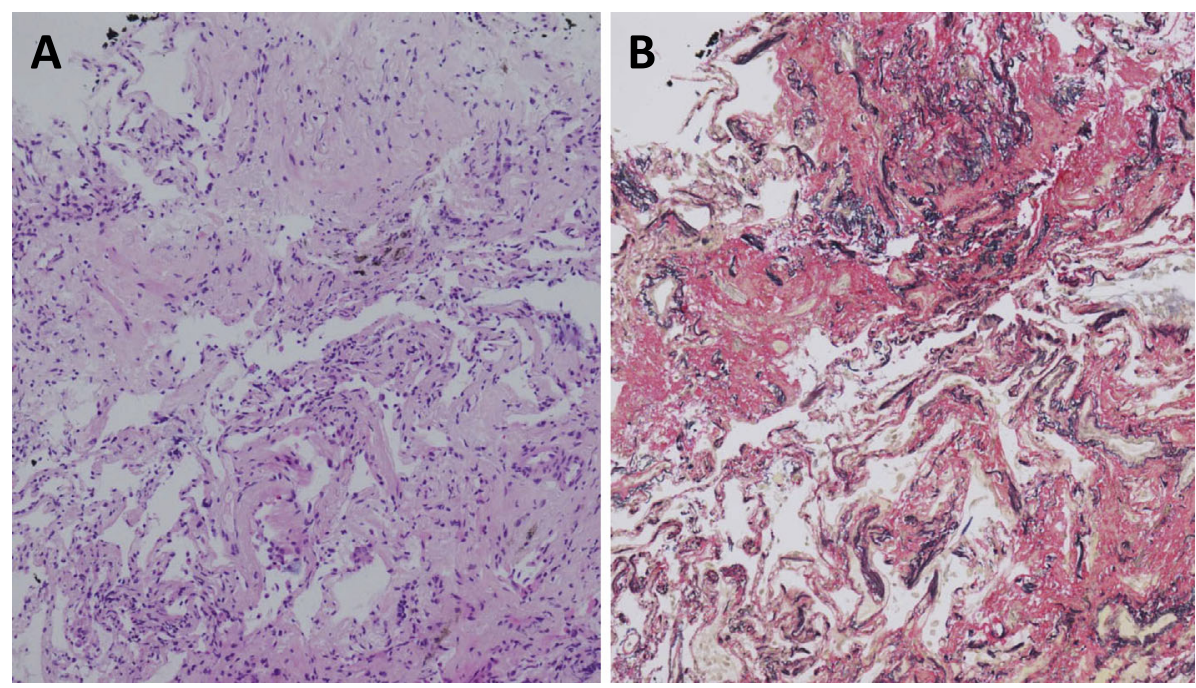

Figure 4. The pathological findings of a transbronchial lung biopsy showed airspace organization [(A) Hematoxylin and Eosin staining and (B) Elastica van Gieson staining; ×50 magnification].

Plasma vascular endothelial growth factor (VEGF) was markedly increased to $368 \mathrm{pg} / \mathrm{mL}$ (normal: $<38.3 \mathrm{pg} / \mathrm{mL}$ ).

Transthoracic echocardiography showed a normal left ventricular function. A rapid influenza diagnostic test and Mycoplasma antigen test using nasopharyngeal swabs and a blood culture were all negative. We suspected him of having RS3PE syndrome due to the acute onset of arthritis and edema. X-ray and gadolinium contrast-enhanced dynamic magnetic resonance imaging of the patient's hands showed soft tissue swelling and extensor tenosynovitis without bone erosions (Fig. 3). Our patient met the diagnostic criteria of RS3PE syndrome, including 1) pitting edema of the hands and feet, 2) a sudden onset of polyarthritis, 3) an onset at age $\geq 50$ years old, and 4) negative serology for rheumatoid factors (1). HRCT revealed consolidation on a background of lung fibrosis (Fig. 1D-F).

Bronchoalveolar lavage (BAL) fluid obtained from the medial segmental bronchus showed a total cell count of $1.9 \times$ $10^{5}$ cells/mL (macrophages, $86.0 \%$; lymphocytes, $6.7 \%$; neutrophils, $6.5 \%$; and eosinophils, $0.8 \%$ ). No significant pathogens or viruses were cultured or detected by multiplex realtime polymerase chain reaction (FTD Resp 21 Kit; Fast Track Diagnostics, Silema, Malta) from the BAL fluid. A transbronchial lung biopsy specimen revealed intraluminal organization (Fig. 4). We considered these pulmonary lesions to be a complication of RS3PE syndrome because of the concomitant onset of pulmonary and extrapulmonary lesions. 
A high-grade fever persisted despite the administration of antibiotics. Prednisolone $20 \mathrm{mg}$ was initiated from hospital day 6 , after which his fever, edema, polyarthritis, and respiratory failure rapidly improved. His serum KL-6 level decreased slightly to $653 \mathrm{U} / \mathrm{mL}$. Furthermore, the findings from HRCT performed on hospital day 17 confirmed this improvement (Fig. 1G-I). He was discharged on hospital day 20 and continues to be followed on an outpatient basis. He underwent ${ }^{18} \mathrm{~F}$-fluorodeoxyglucose-positron emission tomography/CT and esophagogastroduodenoscopy to investigate potential underlying malignancy, but no evidence of malignancy was found. Parvovirus $\operatorname{IgM}$ and $\operatorname{IgG}$ antibodies were not elevated during the follow-up period.

At 5 months after discharge, his MMP-3 level had fallen to $125.9 \mathrm{ng} / \mathrm{mL}$, and the patient has experienced no recurrence of either pulmonary or extra-pulmonary lesions while continuing treatment with prednisolone at $8 \mathrm{mg}$ per day.

\section{Discussion}

We experienced a rare case of RS3PE syndrome that developed in a patient who had been diagnosed with IPF. Pulmonary consolidation on a background of lung fibrosis appeared concurrently with the onset of RS3PE syndrome, and both the pulmonary lesions and the edema and polyarthritis responded well to steroid therapy.

RS3PE syndrome is a rare inflammatory arthritis that is characterized by 1) a good prognosis, i.e. remission; 2) seronegativity for antibodies, including rheumatoid factors; 3) symmetry; and 4) synovitis with pitting edema of the dorsum of the hands and feet (1). Other common characteristics of RS3PE syndrome include an acute onset in older persons, the absence of bone erosion, inflammatory findings on blood examinations, and painless limitation of movement of the wrist and fingers $(2,3)$. Our patient had an acute onset of bilateral shoulder pain and edema on the dorsum of both feet at the age of 62. These symptoms were resolved by treatment with medium-dose corticosteroid. The diagnosis of RS3PE syndrome was confirmed by these characteristics that were compatible with RS3PE syndrome and the exclusion of other causes of acute multiple joint arthritis, such as rheumatoid arthritis, systemic rheumatic diseases, reactive arthritis, and viral infectious arthritis.

The etiology of RS3PE syndrome is not fully understood. This syndrome has been associated with polymyalgia rheumatica, Parkinson's disease, Sjögren's syndrome, and ankylosing spondylitis, and it has also been considered to be a paraneoplastic rheumatic disease (10-14). To our knowledge, there are no other autoimmune diseases or malignancies that can induce RS3PE syndrome. Specific human leukocyte antigen (HLA) has been associated with RS3PE syndrome (1); however, a clear association with a particular HLA antigen in pulmonary involvement is still lacking. HLA typing was not performed in our patient.

In our patient, consolidation on a background of lung fibrosis appeared concurrently with the polyarthritis and edema. The radiological findings did not improve despite the administration of antibiotics, and the findings of cultures and multiplex real-time polymerase chain reaction from BAL fluid were all negative, suggesting a noninfectious etiology. The brain natriuretic peptide level and echocardiography ruled out cardiogenic pulmonary edema. In addition, both the pulmonary lesions and the edema, polyarthritis, and serum MMP-3 level responded well to steroid therapy. We therefore considered these pulmonary lesions to be a pulmonary complication of RS3PE syndrome.

To our knowledge, there have been only two reported cases of RS3PE syndrome associated with interstitial pneumonia $(7,8)$. The diagnosis in one case was bronchiolitis obliterans organizing pneumonia (OP), and pulmonary lesions were detected concomitantly with the onset of RS3PE syndrome, as in our patient. In the other case, interstitial pneumonia accompanied by severe acute respiratory failure developed after the improvement of RS3PE syndrome, and a diagnosis of OP was made by a transbronchial biopsy after the improvement of respiratory failure with steroid treatment. In the first case (7) and our present case, the pulmonary lesions appeared to be associated with RS3PE syndrome because of the concomitant onset of pulmonary involvement and RS3PE syndrome that responded favorably to corticosteroid therapy. The pulmonary specimen obtained in this case showed histologic changes consistent with a diagnosis of OP. Both of the reported cases also had an OP pattern on pathological findings, suggesting that the OP pattern may be a common histologic manifestation of RS3PE syndrome. Although KL-6 has been suggested to be a good marker for disease activity in interstitial lung diseases associated with systemic autoimmune disorders $(15,16)$, serum KL-6 did not reflect the lung disease activity of RS3PE syndrome in our patient. We considered the results compatible with those of a previous report showing that elevated levels of KL-6 were observed in only half of the patients with cryptogenic OP (17).

Previous studies have suggested that VEGF may contribute to the pathological changes; both synovial hypervascularity (synovitis) and an increment in vascular permeability (subcutaneous edema) may be facilitated by VEGF in patients with RS3PE syndrome (18). Several reports of pleural effusion in patients with RS3PE syndrome have been published (19-21). Among them, the pleural effusion was turbid and exudative in five patients, and one patient showed a high level of serum VEGF. Elevated VEGF levels may be associated with the cause of pleural and pericardial effusion in RS3PE syndrome. The plasma VEGF in our patient was also markedly elevated. Taken together, these present and previous findings suggest that a high VEGF level may induce both synovitis and pulmonary involvement. The same autoantigen in both the synovium and the lung may be recognized by helper $\mathrm{T}$ cells, thus facilitating the simultaneous development of polyarthritis and pulmonary lesions; however, further studies are needed to clarify this issue.

Patients treated for RS3PE syndrome without concomitant 
malignancy have been reported to respond well to low-dose corticosteroids and have a good prognosis (1-5). However, a recent study found that the outcomes of RS3PE syndrome appeared to be worse than previously thought, and some patients required disease-modifying anti-rheumatic drugs (14). Although our patient has not relapsed, careful long-term follow-up for signs of any relapse of RS3PE syndrome will be necessary.

In conclusion, we encountered a case of RS3PE syndrome in a patient with IPF who presented with pulmonary involvement and synovitis. The clinical picture of RS3PE syndrome can include multi-organ involvement, and recognition of the various manifestations of RS3PE syndrome is necessary in order to provide an appropriate diagnosis and carry out effective disease management.

The authors state that they have no Conflict of Interest (COI).

\section{References}

1. McCarty DJ, O'Duffy JD, Pearson L, Hunter JB. Remitting seronegative symmetrical synovitis with pitting edema. RS3PE syndrome. JAMA 254: 2763-2767, 1985.

2. Russell EB, Hunter JB, Pearson L, McCarty DJ. Remitting, seronegative, symmetrical synovitis with pitting edema-13 additional cases. J Rheumatol 17: 633-639, 1990.

3. Olivé A, del Blanco J, Pons M, Vaquero M, Tena X. The clinical spectrum of remitting seronegative symmetrical synovitis with pitting edema. The Catalán Group for the Study of RS3PE. J Rheumatol 24: 333-336, 1997.

4. Chaouat D, Le Parc JM. The syndrome of seronegative symmetrical synovitis with pitting edema (RS3 PE syndrome): a unique form of arthritis in the elderly? Report of 4 additional cases. J Rheumatol 16: 1211-1213, 1989.

5. Sattar MA. Remitting seronegative symmetrical synovitis with pitting oedema in young adults: a subset of rheumatoid arthritis or a distinct syndrome? Br J Rheumatol 29: 479-481, 1990.

6. Bhakta BB, Pease CT. Late-onset rheumatoid arthritis: is pitting oedema of the hands at onset a good prognostic indicator? $\mathrm{Br} \mathrm{J}$ Rheumatol 36: 214-219, 1997.

7. Kato T, Ubara Y, Sawa N, et al. An abrupt onset of seropositive polyarthritis with prominent distal tenosynovitis concomitant with bronochiolitis obliterans organizing pneumonia (BOOP): consideration of the relationship with RS3PE syndrome. Intern Med $\mathbf{4 3}$ : 143-147, 2004.

8. Okuda M, Kashio M, Aitani M, Nakanishi D, Tanaka N, Kimura $\mathrm{K}$. A case of interstitial pneumonia complicating RS3PE syndrome in which soluble interleukin-2 receptor (sIL-2R) proved useful for assessing symptoms. Nihon Kokyuki Gakkai Zasshi (Journal of Japanese Society of Respiratory Medicine) 47: 636-640, 2009 (in
Japanese, Abstract in English).

9. Raghu G, Remy-Jardin M, Myers JL, et al.; American Thoracic Society, European Respiratory Society, Japanese Respiratory Society, Latin American Thoracic Society. Diagnosis of Idiopathic Pulmonary Fibrosis. An Official ATS/ERS/JRS/ALAT Clinical Practice Guideline. Am J Respir Crit Care Med 198: e44-e68, 2018.

10. Russell EB. Remitting seronegative symmetrical synovitis with pitting edema syndrome: followup for neoplasia. J Rheumatol 32: 1760-1761, 2005.

11. Sibilia J, Friess S, Schaeverbeke T, et al. Remitting seronegative symmetrical synovitis with pitting edema (RS3PE): a form of paraneoplastic polyarthritis? J Rheumatol 26: 115-120, 1999.

12. Choi YM, Sheen DH, Lee YJ, Lee EB, Song YW. Sjögren's syndrome presenting as remitting seronegative symmetric synovitis with pitting edema (RS3PE). J Korean Med Sci 18: 606-608, 2003.

13. Koeger AC, Karmochkine M, Chaïbi P. RS3PE syndrome associated with advanced ankylosing spondylitis. J. Rheumatol 22: 375376, 1995.

14. Ito $\mathrm{E}$, Okamoto $\mathrm{H}$, Mochizuki A, et al. Remitting seronegative symmetrical synovitis with pitting edema (RS3PE) syndrome accompanied by Parkinson's disease. Intern Med 46: 1139-1142, 2007.

15. Kumanovics G, Minier T, Radics J, Palinkas L, Berki T, Czirjak L. Comprehensive investigation of novel serum markers of pulmonary fibrosis associated with systemic sclerosis and dermato/polymyositis. Clin Exp Rheumatol 26: 414-420, 2008.

16. Bandoh S, Fujita J, Ohtsuki Y, et al. Sequential changes of KL-6 in sera of patients with interstitial pneumonia associated with polymyositis/dermatomyositis. Ann Rheum Dis 59: 257-262, 2000.

17. Okada F, Ando Y, Honda K, Tanoue S, Matsumoto S, Mori H. Comparison of pulmonary CT findings and serum KL-6 levels in patients with cryptogenic organizing pneumonia. Br J Radiol 82: 212-218, 2009.

18. Arima $\mathrm{K}$, Origuchi $\mathrm{T}$, Tamai $\mathrm{M}$, et al. RS3PE syndrome presenting as vascular endothelial growth factor associated disorder. Ann Rheum Dis 64: 1653-1655, 2005.

19. Yanamoto S, Fukae J, Fukiyama Y, Fujioka S, Ouma S, Tsuboi Y. Idiopathic remitting seronegative symmetrical synovitis with pitting edema syndrome associated with bilateral pleural and pericardial effusions: a case report. J Med Case Rep 10: 198, 2016.

20. Oide T, Ohara $S$, Oguchi K, et al. Remitting seronegative symmetrical synovitis with pitting edema (RS3PE) syndrome in Nagano, Japan: clinical, radiological, and cytokine studies of 13 patients. Clin Exp Rheumatol 22: 91-98, 2004.

21. Matsunaga T, Izumi Y, Iwanaga $\mathrm{N}$, et al. Myelodysplastic syndrome precedes the onset of remitting seronegative symmetrical synovitis with pitting edema (RS3PE) syndrome. Tohoku J Exp Med 235: 47-52, 2015.

The Internal Medicine is an Open Access journal distributed under the Creative Commons Attribution-NonCommercial-NoDerivatives 4.0 International License. To view the details of this license, please visit (https://creativecommons.org/licenses/ by-nc-nd/4.0/).

\section{(C) 2020 The Japanese Society of Internal Medicine} Intern Med 59: 1065-1069, 2020 